\title{
Agronomic characterization of sweet potato accessions
}

\author{
Pablo Forlan Vargas'*, Dumon Ramiro Zanetti Godoy², \\ Luis Carlos Ferreira de Almeida' ${ }^{2}$, Renata Castoldi ${ }^{3}$ \\ 'São Paulo State University, Botucatu, SP, Brazi

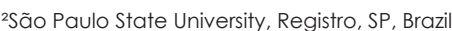 \\ ${ }^{3}$ Federal University of Uberlândia, Uberlândia, MG, Brazi \\ *Corresponding author, e-mail: pablo@registro.unesp.br
}

\begin{abstract}
The agronomic characterization of a genotype set is one of the basic steps to start a breeding program, as action strategies to search for new cultivars are defined through the knowledge of these characteristics. Thus, the aim of this study was to rescue and assess agronomic performance in accessions collected in traditional communities. Sweet potatoes were collected among "quilombos", indigenous villages, native populations and small farmers from Vale do Ribeira. The study was conducted at UNESP, Registro Campus, from February/2013 to August/2014. The experiment was set in a randomized blocks design with 95 accessions, two commercial cultivars, Brazlândia Branca and Brazlândia Roxa (controls), with three repetitions. Total commercial roots production, percentage of commercial roots production, total roots dry matter production, roots dry matter percentage, fresh branches production, total dry matter production in branches and dry matter percentage in branches were assessed. Accessions VR13-62, VR13-61, VR13-44, VR13-11 and VR 13-14 demonstrated characteristics of roots for consumption and VR 13-11 and VR 13-14 for industry consumption due to the high dry matter percentage obtained per hectare; VR13-35, VR13-87 and VR13-22 are adequate for animal feed purposes. VR13-11, VR13-22 and VR13-58 accessions can be used for human and animal consumption; VR 13-48 was suitable for home and industry consumption; and VR13-4 and VR13-31 was suitable for animal feed and industry consumption.
\end{abstract}

Keywords: agronomic performance, germplasm, Ipomoea batatas, pre-breeding

\section{Caracterização agronômica de acessos de batata-doce}

\section{Resumo}

A caracterização agronômica de um conjunto de genótipos é uma das etapas fundamentais no início de um programa de melhoramento, haja vista que a partir do conhecimento dessas características, define-se estratégias de ação na busca de novas cultivares. Desta forma, objetivouse avaliar agronomicamente acessos coletados em comunidades tradicionais. Foram realizadas coletas de batatas-doces em quilombos, aldeias indígenas, caiçaras e pequenos agricultores do Vale do Ribeira. A condução do estudo foi realizada em campo da Unesp-Campus de Registro, entre fevereiro/2013 e agosto/2014. O delineamento experimental foi em blocos casualizados, com 95 acessos coletados e duas cultivares comerciais, Brazlândia Branca e Roxa (testemunhas), com três repetições. Foram avaliadas as características: produção total e comercial de raiz, percentagem de produção comercial de raízes, produção total de massa seca das raízes, percentagem de massa seca nas raízes, produção de ramas frescas, produção total de matéria seca nas ramas e percentagem de matéria seca nas ramas. Verificou-se que os acessos VR13-62, VR13-61, VR1344, VR13-11 e VR13-14 são promissores para produção de raízes para consumo domiciliar; VR1311 e VR13-14 são promissores para indústria; e, VR13-35, VR13-87 e VR13-82 são promissores para produção de ramas para alimentação animal. Os acessos VR13-11, VR13-22 e VR13-58 possuem dupla aptidão para consumo domiciliar e animal; VR 13-48 para consumo domiciliar e para indústria; e, VR13-4 e VR13-31 para o consumo animal e indústria.

Palavras-chave: desempenho agronômico, germoplasma, Ipomoea batatas, pré-melhoramento 


\section{Introduction}

Sweet potato is among the world's seven most consumed foods. It is considered capable of ensuring the food security for population, especially the low-income populations, due to the amount produced and the quality of the vegetable.

With the change of population's consumption habits, especially in traditional communities (which have been increasing the consumption of processed foods), it is possible to observe a decrease in sweet potato consumption. Therefore, many sweet potato landraces and their genetic diversity are disappearing.

At the end of the decade of 1970, the annual production of sweet potato in Brazil was 819,000 tons. After two decadesr, the production decreased $42 \%$, with the amount of 479,000 tons. In the same period, Argentina increased its production by $25.77 \%$ (FAO, 2015), even though Brazil has areas with better climate and soil conditions when compared to other countries.

In 2013, the Brazilian States with higher production were Rio Grande do Sul 1166,600 tons), Sao Paulo (71,400 tons), Sergipe (44,300 tons), Minas Gerais $(30,100$ tons) and Paraná (30,800 tons). The highest yield (38.8 tons per hectare) was obtained in Mato Grosso, with 221 hectares (Anuário Brasileiro de Hortaliças, 2015).

Although it is an important crop for the country, mainly due to its social function, sweet potato has been little studied in Brazil, especially with regard to the development of new productive cultivars adapted to different regions of the country (Massaroto et al., 2014).

Sweet potato roots are rich in both energy and nutrients. It is a target food in studies from several countries due to its high content of carotenoids and flavonoids.

Sweet potato is traditionally used as human food, although it is commonly used in animal feed (Pedrosa et al., 2015; Khalid et al., 2013), in which it can be supplied in fresh or ensiled form (Andrade Junior et al., 2014).

In countries like China and Vietnam, branches (used exclusively or combined with roots) are widely used to feed pigs. In Brazil however, the use of sweet potato branches in animal feed is only made on a limited scale. It is assumed that most of branches are discarded as a residue residue (Monteiro et al., 2007).

Sweet potato has also great potential for ethanol production in Brazil (Gonçalves Neto et al., 2011) and 150 liters of ethanol per ton of root can be produced (Machado \& Abreu, 2007), while sugarcane can produce 80 liters per ton (Ramos, 2007).

Agronomic characterization of a genotype set is an essential step in a breeding program, as action strategies to search for new cultivars are defined through the knowledge of these characteristics.

Under the hypothesis that there is agronomic performance variability among Ipomoea batatas accessions growed by farmers, the aim of this study was to rescue and characterize agronomically the accessions collected in traditional communities from Vale do Ribeira, Brazil.

\section{Material and Methods}

Samples were collected in traditional communities from Vale do Ribeira: "quilombos", indigenous villages and native populations. Accessions were also collected from small farmers in peri-urban plantations where sweet potato has been grown for subsistence, between February and November, 2013.

In each collection point, municipality, site (community or neighborhood description), donor's name and geographical coordinates (Figure 1) were identified. Identification/code was given taking into consideration the region, year and collection number.

Young branches of sweet potatoes were collected, and in absence of branches, the roots were collected. The material was identified, stored in paper bags and transported to Unesp, Registro Campus. Ninety-five sweet potato accessions were collected in seven municipalities from Vale do Ribeira, contemplating the Upper, Middle and Lower region.

The evaluation of the accessions was conducted at UNESP, Registro Campus, located in $24^{\circ} 32^{\prime} 05^{\prime \prime} S$ and $47^{\circ} 51^{\prime} 40^{\prime \prime} \mathrm{W}$, from February to August, 2014.

According to Köppen classification, the climate of the region is Cfa (Cepagri, 2015), with 
hot summers and rainfall concentration during summer, without a defined dry season. In Figure 2 is possible to observe the rainfall and temperature averages data during the studied period.

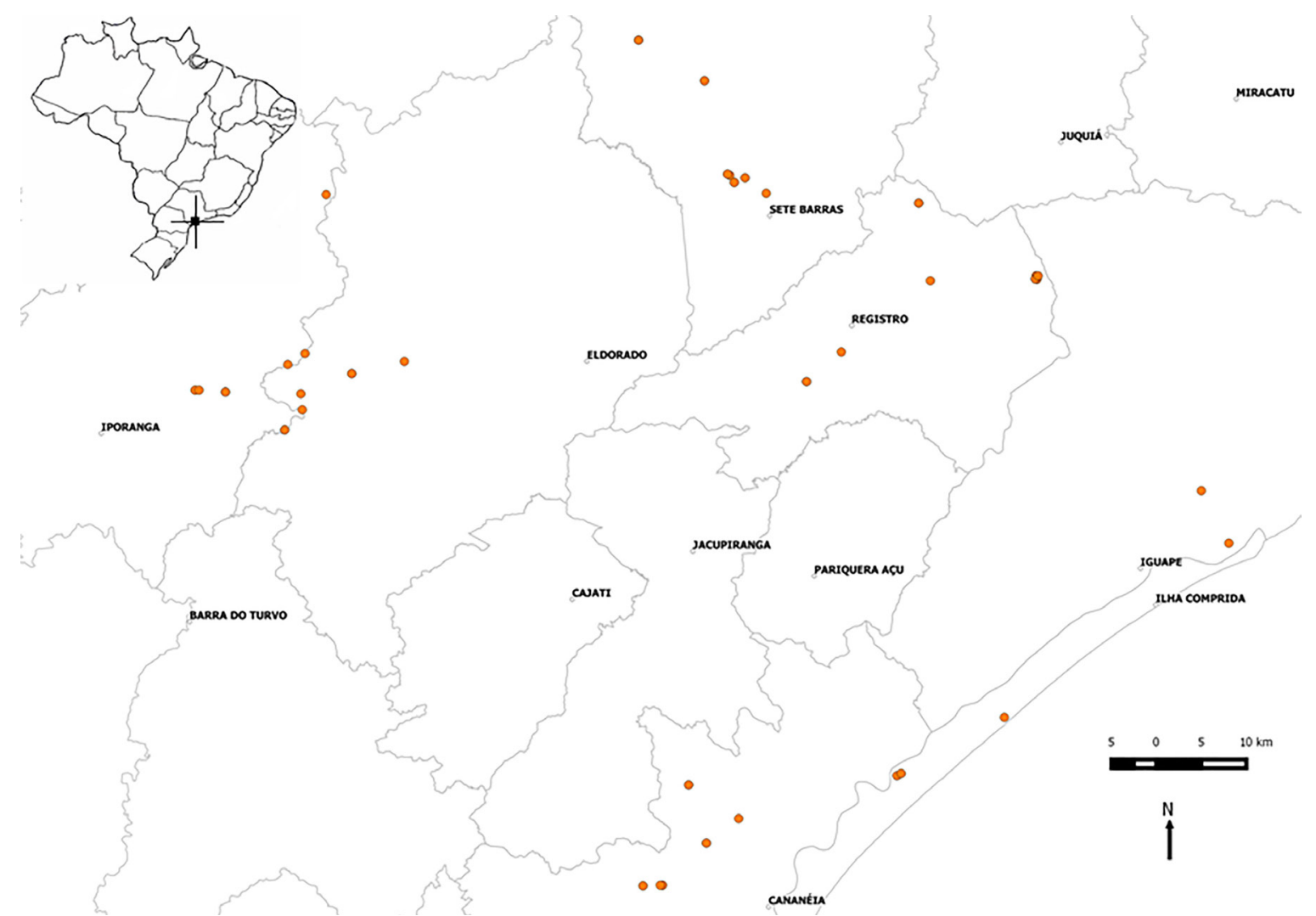

Figure 1. Sweet potato accessions points in Vale do Ribeira, SP, Brazil.

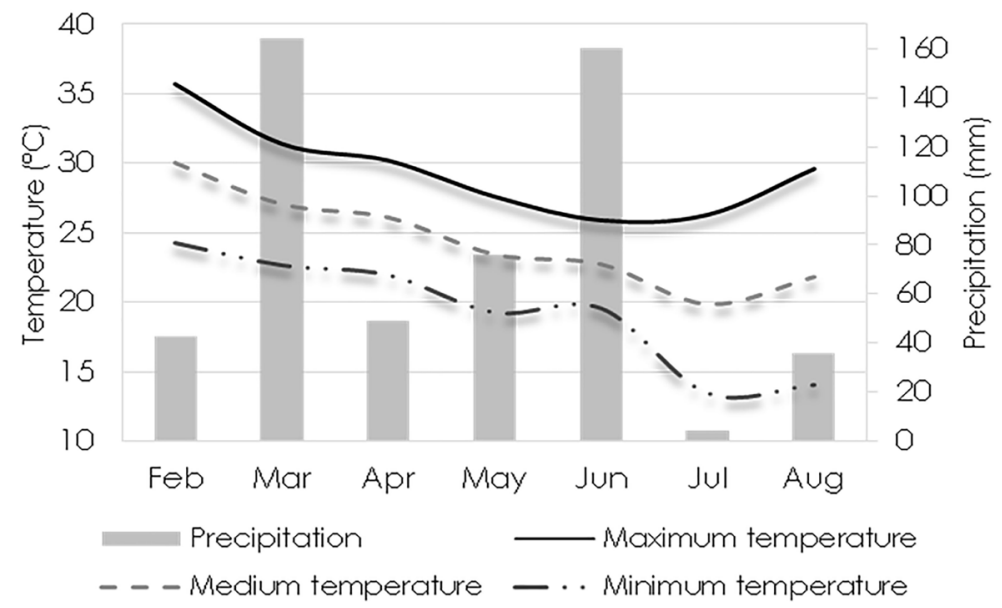

Figure 2. Climatic data obtained at the weather station located at UNESP experimental farm, Registro Campus, from February to August, 2014.

The experiment was conducted in a randomized block design, with 95 accessions collected in Vale do Ribeira and two cultivars - Brazlândia Branca and Brazlândia Roxa - as controls. Thus, the study consisted of 97 treatments with three repetitions. Each experimental plot contained 10 plants in a single row, and the viable samples was comprised by eight central plants.
The cultivar Brazlândia Branca is characterized by white skin and light cream flesh. The root shape is elongated and uniform with large leaves, measuring from $12 \times 14$ to $15 \times 17 \mathrm{~cm}$ (Embrapa, 2015).

The cultivar Brazlândia Roxa characterized by purple skin and cream flesh. The shape of the root is elongated and uniform. Leaves (old and young) are green with $11 \times 10$ to 
$15 \times 15 \mathrm{~cm}$ (EMBRAPA, 2015).

Soil chemical properties were measured before the study, through soil samples collected in the 0-20 cm deep layer. Samples were sent to the Soil Fertility Laboratory of Unesp-Botucatu Campus.

According to soil chemical analysis, the following results were obtained: $\mathrm{pH}\left(\mathrm{CaCl}_{2}\right)$ of 5.0; Organic matter of $18 \mathrm{~g} \mathrm{dm}^{-3} ; \mathrm{P}$ (resin) and $\mathrm{S}$ of 2 and $3 \mathrm{mg} \mathrm{dm}^{-3}$, respectively; $\mathrm{K}, \mathrm{Ca}, \mathrm{Mg}, \mathrm{Al}$ and $\mathrm{Al}+\mathrm{H}$ of $0.5,16.0,10.0,0.0$ and $24.0 \mathrm{mmol}_{\mathrm{c}} \mathrm{dm}^{-3}$, respectively; $\mathrm{B}, \mathrm{CU}, \mathrm{Fe}, \mathrm{Mn}$ and $\mathrm{Zn}$ of $0.16,1.8$, 32.0, 0.5 and $0.9 \mathrm{mg} \mathrm{dm}^{-3}$, respectively; Cation Exchange Capacity of 51 and $53 \%$ of base saturation.

Liming was held two months before the transplantation, in order to raise the base saturation to $60 \%$ (Monteiro \& Peressin, 1997). The amount of $0.396 \mathrm{tha}^{-1}$ of dolomitic limestone, PRNT 90\%, was manually applied in the area.

On February 1 15t, 2014, each accession was propagated from sweet potato mini-cuttings with two nodes. Mini-cuttings were taken from the middle and upper thirds of the branches. Leaves were removed with pruning shears, and its basal bud was inserted in a substrate with Bioplante ${ }^{\circledR}$ $\mathrm{HA}$ and $\mathrm{HT}+$ cattle manure mix, in a 2:2:1 ratio. Trays with 72 cells $(11 \mathrm{~cm}$ high and $5 \mathrm{~cm}$ wide) of expanded polystyrene were used. The propagation type was chosen due to the limited number of accession branches, besides being a well-established technique (Rós \& Narita, 2011; Rós et al., 201 1), and seedlings were irrigated twice a day. The studied area was prepared twice with plowing followed by harrowing. Subsequently, windrows were manually formed with one meter of distance and $40 \mathrm{~cm}$ high. Simultaneously, the fertilization was conducted with $500 \mathrm{~kg} \mathrm{ha}^{-1}$ of 4:14:8, $133 \mathrm{~kg} \mathrm{ha}^{-1}$ of potassium chloride and 166 $\mathrm{kg} \mathrm{ha}^{-1}$ of single superphosphate, corresponding to 20,100 and $120 \mathrm{~kg} \mathrm{ha}^{-1}$ of nitrogen, phosphorus and potassium (Monteiro \& Peressin, 1997), respectively. The fertilizer was incorporated through windrow turning.

Transplant to the definitive location was conducted on February $24^{\text {th }}$, when seedlings were completely developed. Row spacing of 1 $\mathrm{m}$ and plant spacing of $0.25 \mathrm{~cm}$ were used. Thus, the final stand was set with 40,000 plants per hectare.

On March $14^{\text {th }}$, a fertilization with $66 \mathrm{~kg} \mathrm{ha}^{-1}$ urea was carried out, in order to obtain $30 \mathrm{~kg} \mathrm{ha-1} \mathrm{N}$ (Monteiro \& Peressin, 1997). Branches were "combed" at 30 and 50 days after transplantation, in order to facilitate transit between crop spacing and to avoid branches interlacing. No infestation of pests and deseases were observed. Weed control was conducted manually at 20 and 40 days after transplantation.

On July $27^{\text {th }}$ harvest of roots began, in which the following parameters were assessed:

a) Root total production (RTP): weight of all roots harvested in the plot, later converted to $\mathrm{kg} \mathrm{ha}^{-1}$.

b) Root commercial production (RCP): weight of roots above $80 \mathrm{~g}$ in the plot, later converted to $\mathrm{kg} \mathrm{ha}^{-1}$.

c) Root commercial production percentage (RPP): obtained by the equation: $\mathrm{RCP}=(\mathrm{PCR} / \mathrm{TP}) \times 100$.

d) Root dry matter percentage (RDMP): $2 \mathrm{~kg}$ root samples were dried in a greenhouse at $65^{\circ} \mathrm{C}$ until reaching constant weight, in order to determine the dry matter content (\%).

e) Root total dry matter production (RTDMP): obtained by the equation:

RTDMP $=$ TP $\times$ RDMP.

f) Fresh branches production (FBP): obtained by cutting branches and leaves at soil level, with subsequent plot weighing and conversion to $\mathrm{kg} \mathrm{ha}^{-1}$.

g) Branches dry matter percentage (BDMP): $2 \mathrm{~kg}$ of shoot samples were grounded. Subsequently, they were placed in an oven at $65^{\circ} \mathrm{C}$ until reaching constant weight, in order to determine the dry matter content (\%).

h) Branches total dry matter production (BTDMP): obtained by the equation: RTDMP $=$ FBP $x$ BDMP.

The evaluations were carried out using a digital electronic scale (model Toledo 9094C/5) with $15 \mathrm{~kg} \pm 5 \mathrm{~g}$ of capacity and an electronic scale (model Shimadzu BL3200) with 3,200g \pm $0.01 \mathrm{~g}$ capacity were used.

With average data, Lilliefors test was applied to check data distribution. Subsequently, data were submitted to analysis of variance by $F$ test, and averages were grouped by Skott-Knott 
test at $5 \%$, using the Genes software (Cruz, 2013), 5.1 version.

\section{Results and Discussion}

All characteristics assessed presented normal distribution of errors. There were significant differences for all evaluated characteristics according to $F$ test $(p<0.01)$.

Fifteen genotype groups were formed considering the fresh roots production characteristics and according to Scott-Knott's test at $5 \%$ and the cluster test. As for commercial production percentage, five groups were formed. Values were the highest and lowest for the group formation, respectively, for the eight evaluated agronomic characteristics (Table 1). Thus, high phenotypic variability was observed within the studied population.

Table1. Total branches production (BTP), branches total dry matter production (BTDMP), branches dry matter percentage (BDMP), total root production (RTP), root commercial production (RCP), commercial root percentage (CRP), root dry matter percentage (RDMP) and root total dry matter (RTDM) of sweet potato accessions collected in Vale do Ribeira, Brazil

\begin{tabular}{|c|c|c|c|c|c|c|c|c|}
\hline Accession & $\begin{array}{c}\text { BTP } \\
\left(\mathrm{kg} \mathrm{ha}^{-1}\right)\end{array}$ & $\begin{array}{l}\text { BTDMP } \\
\left(\mathrm{kg} \mathrm{ha-1)}^{-1}\right)\end{array}$ & $\begin{array}{c}\text { BDMP } \\
(\%)\end{array}$ & $\begin{array}{c}\text { RTP } \\
\left(\mathrm{kg} \mathrm{ha}^{-1}\right)\end{array}$ & $\begin{array}{c}\mathrm{RCP} \\
\left(\mathrm{kg} \mathrm{ha}^{-1}\right)\end{array}$ & $\begin{array}{l}\text { CRP } \\
(\%)\end{array}$ & $\begin{array}{l}\text { RDMP } \\
(\%)\end{array}$ & $\begin{array}{c}\text { RTDM } \\
\left(\mathrm{kg} \mathrm{ha}^{-1}\right)\end{array}$ \\
\hline VR13-01 & $22,413.37 \mathrm{~h}^{1}$ & $3,350.40 \mathrm{j}$ & $14.80 \mathrm{C}$ & $22,413.37 \mathrm{~h}^{1}$ & $21,935.15 \mathrm{~g}$ & $97.91 \mathrm{a}$ & $35.52 a$ & $7,962.27 \mathrm{~d}$ \\
\hline VR13-02 & $39,730.35 \mathrm{f}$ & $5,143.91 \mathrm{~h}$ & $13.00 \mathrm{e}$ & $21,012.05 \mathrm{i}$ & $19,593.50 \mathrm{~h}$ & $93.18 \mathrm{~b}$ & $24.29 \mathrm{e}$ & $5,107.60 \mathrm{f}$ \\
\hline VR13-03 & $40,033.00 \mathrm{f}$ & $5,821.63 \mathrm{~g}$ & $14.55 \mathrm{e}$ & $12,716.75 \mid$ & $11,634.17 \mathrm{j}$ & $91.30 \mathrm{~b}$ & $26.67 \mathrm{~d}$ & $3,410.93 \mathrm{~h}$ \\
\hline VR13-04 & $48,350.00 \mathrm{e}$ & $8,205.01 \mathrm{e}$ & $17.23 d$ & $21,769.22 \mathrm{~h}$ & $15,927.56 \mathrm{i}$ & $73.15 \mathrm{c}$ & $33.71 \mathrm{~b}$ & $7,337.14 \mathrm{~d}$ \\
\hline VR13-05 & $19,650.00 \mathrm{i}$ & $3,915.63 \mathrm{i}$ & $19.76 \mathrm{C}$ & $20,405.25 \mathrm{i}$ & $18,907.73 \mathrm{~h}$ & 92.79 b & $36.72 \mathrm{a}$ & $7,494.65 \mathrm{~d}$ \\
\hline VR13-06 & $40,900.00 \mathrm{f}$ & $5,660.36 \mathrm{~g}$ & $13.87 \mathrm{e}$ & $22,371.22 \mathrm{~h}$ & $20,238.37 \mathrm{~g}$ & $90.32 \mathrm{~b}$ & $34.62 \mathrm{~b}$ & $7,740.25 \mathrm{~d}$ \\
\hline VR13-07 & $28,100.00 \mathrm{~g}$ & $4,360.50 i$ & $16.00 \mathrm{~d}$ & $27,403.02 f$ & $27,234.87$ e & $99.39 \mathrm{a}$ & $27.82 d$ & $7,628.30 \mathrm{~d}$ \\
\hline VR13-08 & $41,642.50 \mathrm{f}$ & $5,875.32 \mathrm{~g}$ & $14.17 \mathrm{e}$ & $10,628.90 \mathrm{~m}$ & $9,181.24$ j & $86.71 \mathrm{~b}$ & $30.22 \mathrm{c}$ & $3,217.80 \mathrm{~h}$ \\
\hline VR13-09 & $43,925.00 \mathrm{f}$ & $6,076.85 \mathrm{~g}$ & $13.96 \mathrm{e}$ & $17,459.57$ j & $17,009.22 \mathrm{~h}$ & $97.49 \mathrm{a}$ & $32.70 \mathrm{~b}$ & $5,705.37 f$ \\
\hline VR13-10 & $29,885.50 \mathrm{~g}$ & $4,262.25 \mathrm{i}$ & $14.50 \mathrm{e}$ & $19,827.52 i$ & $18,796.70 \mathrm{~h}$ & $94.90 \mathrm{a}$ & $30.21 \mathrm{c}$ & $6,022.97 \mathrm{e}$ \\
\hline VR13-11 & $65,300.00 \mathrm{~d}$ & $7,139.66 \mathrm{f}$ & $11.00 \mathrm{f}$ & $44,636.25 b$ & $43,220.42 \mathrm{~b}$ & $96.83 a$ & $27.82 \mathrm{~d}$ & $12,405.39 a$ \\
\hline VR13-12 & $49,275.00 \mathrm{e}$ & $5,668.87 \mathrm{~g}$ & $11.50 \mathrm{f}$ & $25,554.55 \mathrm{~g}$ & $24,133.97 \mathrm{f}$ & $94.42 \mathrm{a}$ & $30.80 \mathrm{c}$ & $7,850.07 \mathrm{~d}$ \\
\hline VR13-13 & $28,712.50 \mathrm{~g}$ & $4,606.52 \mathrm{i}$ & $16.50 \mathrm{~d}$ & $17,400.57 j$ & $14,758.45 \mathrm{i}$ & & $34.54 \mathrm{~b}$ & $7.34 \mathrm{e}$ \\
\hline VR13-14 & $28,125.00 \mathrm{~g}$ & $5,253.16 \mathrm{~h}$ & $18.00 \mathrm{c}$ & $38,208.80 \mathrm{c}$ & $37,685.27 c$ & $98.62 \mathrm{a}$ & $31.92 \mathrm{~b}$ & $12,200.19 a$ \\
\hline VR13-15 & $14,650.00 i$ & $2,065.41 \mathrm{j}$ & $14.10 \mathrm{e}$ & $15,627.90 j$ & $15,244.07 i$ & $97.56 a$ & $29.24 \mathrm{C}$ & $5.06 \mathrm{~g}$ \\
\hline VR13-16 & $20,255.00 i$ & $3,926.75 \mathrm{i}$ & $19.41 \mathrm{C}$ & $25,769.97 \mathrm{~g}$ & $25,366.25 f$ & $98.31 \mathrm{a}$ & $34.54 \mathrm{~b}$ & $21 \mathrm{c}$ \\
\hline VR13-17 & $25,075.00 \mathrm{~h}$ & $3,747.87$ i & $15.01 \mathrm{~d}$ & $22,071.40 \mathrm{~h}$ & $21,584.90 \mathrm{~g}$ & $97.74 \mathrm{a}$ & $30.02 \mathrm{c}$ & $6,616.33 \mathrm{e}$ \\
\hline VR13-18 & $25,700.00 \mathrm{~h}$ & $3,467.83 j$ & $13.50 \mathrm{e}$ & $9,882.67 \mathrm{~m}$ & $6,019.63 k$ & $61.17 d$ & $29.90 \mathrm{C}$ & $2,978.02 \mathrm{~h}$ \\
\hline VR13-19 & $22,767.50 \mathrm{~h}$ & $4,464.74 \mathrm{i}$ & $19.84 \mathrm{C}$ & $26,587.02 \mathrm{~g}$ & $25,937.50 \mathrm{f}$ & ta & $25.92 \mathrm{e}$ & $05 \mathrm{e}$ \\
\hline VR13-20 & $25,787.50 \mathrm{~h}$ & $4,994.38 \mathrm{~h}$ & $19.38 \mathrm{c}$ & $15,435.02 j$ & $13,601.00 \mathrm{i}$ & $87.95 b$ & $33.58 \mathrm{~b}$ & $5,186.90 \mathrm{f}$ \\
\hline VR13-21 & $18,100.00 i$ & $2,563.40 \mathrm{j}$ & $14.40 \mathrm{e}$ & $23,303.35 \mathrm{~h}$ & $22,553.45 \mathrm{~g}$ & $96.81 \mathrm{a}$ & $29.59 \mathrm{C}$ & $6,886.23 \mathrm{e}$ \\
\hline VR13-22 & $88,250.00 \mathrm{~b}$ & $12,355.00 \mathrm{c}$ & $14.00 \mathrm{e}$ & $35,367.85$ & $33,813.00 \mathrm{~d}$ & $95.53 \mathrm{a}$ & $29.83 c$ & 10,53 \\
\hline VR13-23 & $22,900.00 \mathrm{~h}$ & $3,810.53 \mathrm{i}$ & $16.67 \mathrm{~d}$ & $13,655.60 \mathrm{k}$ & $10,625.50 \mathrm{j}$ & $77.50 \mathrm{c}$ & $34.90 \mathrm{a}$ & $4,719.26 \mathrm{~g}$ \\
\hline VR13-24 & $47,600.00 \mathrm{e}$ & $5,236.00 \mathrm{~h}$ & $11.00 \mathrm{f}$ & $26,244.04 \mathrm{~g}$ & $24,672.02 f$ & $94.27 a$ & $30.72 \mathrm{c}$ & $8,105.82 d$ \\
\hline VR13-25 & $25,737.50 \mathrm{~h}$ & $3,211.81 \mathrm{j}$ & $12.50 \mathrm{f}$ & $14,828.17 \mathrm{k}$ & $13,779.25 \mathrm{i}$ & $92.92 \mathrm{~b}$ & $31.71 \mathrm{~b}$ & $4,701.72 \mathrm{~g}$ \\
\hline VR13-26 & $29,112.50 \mathrm{~g}$ & $4,250.93 \mathrm{i}$ & $14.50 \mathrm{e}$ & $19,930.12 \mathrm{i}$ & $19,398.00 \mathrm{~h}$ & $97.33 \mathrm{a}$ & $30.50 \mathrm{c}$ & $6,079.96 \mathrm{e}$ \\
\hline VR13-27 & $23,887.50 \mathrm{~h}$ & $4,512.23 i$ & $19.05 \mathrm{C}$ & $25,747.92 \mathrm{~g}$ & $25,484.97 \mathrm{f}$ & $99.09 \mathrm{a}$ & $33.75 \mathrm{~b}$ & $8,608.75 \mathrm{c}$ \\
\hline VR13-28 & $31,151.78 \mathrm{~g}$ & $5,333.45 \mathrm{~h}$ & $17.23 d$ & $34,780.45 \mathrm{~d}$ & $33,313.50 \mathrm{~d}$ & $95.71 \mathrm{a}$ & $28.68 d$ & $9,977.77 \mathrm{~b}$ \\
\hline VR13-29 & $32,982.14 \mathrm{~g}$ & $3,922.31 \mathrm{i}$ & $12.00 \mathrm{f}$ & $20,121.67 \mathrm{i}$ & $19,038.22 \mathrm{~h}$ & $94.56 \mathrm{a}$ & $31.83 \mathrm{~b}$ & $6,402.09 \mathrm{e}$ \\
\hline VR13-30 & $22,950.00 \mathrm{~h}$ & $4,338.70 \mathrm{i}$ & $18.73 \mathrm{C}$ & $11,759.75 \mid$ & $10,589.02 j$ & $89.75 b$ & $32.40 \mathrm{~b}$ & $3,803.63 \mathrm{~h}$ \\
\hline VR13-31 & $48,837.50 \mathrm{e}$ & $7,900.48 f$ & $16.26 \mathrm{~d}$ & $27,901.22 \mathrm{f}$ & $26,720.50 \mathrm{e}$ & $95.56 \mathrm{a}$ & $34.25 \mathrm{~b}$ & $9,572.93 b$ \\
\hline VR13-32 & $23,837.50 \mathrm{~h}$ & $3,009.15$ j & $12.52 \mathrm{f}$ & $22,753.12$ & $20,397.97 \mathrm{~g}$ & 89.78 b & $34.50 \mathrm{~b}$ & $7,845.11 \mathrm{~d}$ \\
\hline VR13-33 & $26,162.50 \mathrm{~h}$ & $3,822.86 \mathrm{i}$ & $14.51 \mathrm{e}$ & $11,830.321$ & $10,183.00 \mathrm{j}$ & $85.29 b$ & $31.09 \mathrm{c}$ & $3,679.65 \mathrm{~h}$ \\
\hline VR13-34 & $25,850.00 \mathrm{~h}$ & $3,483.80 \mathrm{j}$ & $13.54 \mathrm{e}$ & $20,482.52 i$ & $20,208.27 \mathrm{~g}$ & $98.65 a$ & $35.55 a$ & $7,283.19 \mathrm{~d}$ \\
\hline VR13-35 & $9,7995.50 \mathrm{a}$ & $13,437.99 \mathrm{~b}$ & $13.80 \mathrm{e}$ & $17,275.27 \mathrm{j}$ & $15,661.00 \mathrm{i}$ & $90.64 \mathrm{~b}$ & $29.83 \mathrm{c}$ & $5,149.43 \mathrm{f}$ \\
\hline VR13-36 & $25,650.00 \mathrm{~h}$ & $3,423.75 \mathrm{j}$ & $13.50 \mathrm{e}$ & $26,441.97 \mathrm{~g}$ & $24,649.00 \mathrm{f}$ & $93.16 \mathrm{~b}$ & $30.28 c$ & $8,017.96 \mathrm{~d}$ \\
\hline VR13-37 & $28,675.00 \mathrm{~g}$ & $5,403.05 \mathrm{~h}$ & $18.81 \mathrm{C}$ & $27,739.30 \mathrm{f}$ & $27,506.18 \mathrm{e}$ & $98.97 \mathrm{a}$ & $35.89 a$ & $10,012.52 \mathrm{~b}$ \\
\hline VR13-38 & $30,142.50 \mathrm{~g}$ & $4,106.66 \mathrm{i}$ & $13.62 \mathrm{e}$ & $19,274.47 i$ & $17,007.92 \mathrm{~h}$ & $88.22 b$ & $31.68 \mathrm{~b}$ & $6,137.42 \mathrm{e}$ \\
\hline VR13-39 & $56,896.00 \mathrm{e}$ & $7,317.60 \mathrm{f}$ & $13.08 \mathrm{e}$ & $19,560.17 i$ & $18,682.49 \mathrm{~h}$ & $94.83 a$ & $28.49 \mathrm{~d}$ & $5,561.23 f$ \\
\hline VR13-40 & $22,735.50 \mathrm{~h}$ & $3,850.81 \mathrm{i}$ & $16.94 \mathrm{~d}$ & $14,562.07 \mathrm{k}$ & $14,008.94 i$ & $96.21 \mathrm{a}$ & $30.33 c$ & $4,426.15 \mathrm{~g}$ \\
\hline VR13-41 & $43,178.50 \mathrm{f}$ & $8,614.74 \mathrm{e}$ & $19.98 \mathrm{C}$ & $35,435.50 \mathrm{~d}$ & $33,943.21 \mathrm{~d}$ & 95.72 a & $25.33 \mathrm{e}$ & $8,953.04 \mathrm{C}$ \\
\hline
\end{tabular}


Vargas et al. (2016) / Agronomic characterization of sweet...

\begin{tabular}{|c|c|c|c|c|c|c|c|c|}
\hline VR13-42 & $67,037.50 \mathrm{~d}$ & $8,860.82 \mathrm{e}$ & $13.32 \mathrm{e}$ & $8,534.75 \mathrm{~m}$ & $7,391.02 \mathrm{k}$ & $86.70 \mathrm{~b}$ & $22.92 \mathrm{e}$ & $1,977.80 \mathrm{i}$ \\
\hline VR13-43 & $72,605.00 \mathrm{c}$ & $7,595.26 f$ & $10.50 \mathrm{f}$ & $20,664.95 \mathrm{i}$ & $20,042.14 \mathrm{~g}$ & $96.70 \mathrm{a}$ & $20.33 f$ & $4,173.10 \mathrm{~g}$ \\
\hline VR13-44 & $32,155.00 \mathrm{~g}$ & $4,037.80 \mathrm{i}$ & $12.58 \mathrm{f}$ & $46,011.12 \mathrm{~b}$ & $44,439.57 \mathrm{~b}$ & $96.59 \mathrm{a}$ & $16.61 \mathrm{~g}$ & $7,662.89 \mathrm{~d}$ \\
\hline VR13-45 & $48,528.50 \mathrm{e}$ & $8,524.59 \mathrm{e}$ & $17.59 \mathrm{~d}$ & $14,362.02 \mathrm{k}$ & $12,797.63 \mathrm{j}$ & $89.05 \mathrm{~b}$ & $32.04 \mathrm{~b}$ & $4,604.09 \mathrm{~g}$ \\
\hline VR13-46 & $27,322.50 \mathrm{~g}$ & $4,775.28 \mathrm{~h}$ & $17.41 \mathrm{~d}$ & 18,368.62 j & $16,713.82 \mathrm{i}$ & $90.25 \mathrm{~b}$ & $30.47 c$ & $5,604.88 f$ \\
\hline VR13-47 & $34,050.00 \mathrm{~g}$ & $6,486.13 \mathrm{~g}$ & $19.04 \mathrm{C}$ & $13,935.15 \mathrm{k}$ & $11,875.72 \mathrm{j}$ & $84.15 b$ & $32.57 \mathrm{~b}$ & $4,539.78 \mathrm{~g}$ \\
\hline VR13-48 & $26,537.50 \mathrm{~h}$ & $4,293.34 \mathrm{i}$ & $16.24 \mathrm{~d}$ & $33,580.15 \mathrm{~d}$ & $32,802.07 \mathrm{~d}$ & $97.60 \mathrm{a}$ & $30.45 c$ & $10,263.89 \mathrm{~b}$ \\
\hline VR13-49 & $11,839.00 \mathrm{i}$ & $2,454.36 \mathrm{j}$ & $20.78 b$ & $14,013.07 \mathrm{k}$ & $12,410.55 \mathrm{j}$ & $86.60 \mathrm{~b}$ & $33.16 \mathrm{~b}$ & $4,665.83 \mathrm{~g}$ \\
\hline VR13-50 & $34,442.50 \mathrm{~g}$ & $4,979.44 \mathrm{~h}$ & $14.50 \mathrm{e}$ & $24,144.47 \mathrm{~h}$ & $23,818.45 f$ & $98.62 \mathrm{a}$ & $31.27 \mathrm{~b}$ & $7,523.45 \mathrm{~d}$ \\
\hline VR13-51 & $32,812.50 \mathrm{~g}$ & $4,666.77 \mathrm{~h}$ & $14.54 \mathrm{e}$ & $29,758.10 \mathrm{f}$ & $28,818.97 \mathrm{e}$ & $96.75 \mathrm{a}$ & $24.50 \mathrm{e}$ & $7,279.13 \mathrm{~d}$ \\
\hline VR13-52 & $43,875.50 \mathrm{f}$ & $5,029.43 \mathrm{~h}$ & $11.50 \mathrm{f}$ & $33,762.67 \mathrm{~d}$ & $31,731.59 \mathrm{~d}$ & $93.92 \mathrm{a}$ & $26.28 \mathrm{e}$ & $8,867.31 \mathrm{C}$ \\
\hline VR13-53 & $21,093.50 \mathrm{~h}$ & $3,113.84 j$ & $14.75 \mathrm{e}$ & $23,462.92 \mathrm{~h}$ & $22,489.22 \mathrm{~g}$ & $95.62 a$ & $31.52 \mathrm{~b}$ & $7,410.33 d$ \\
\hline VR13-54 & $35,845.00 \mathrm{~g}$ & $4,264.34 \mathrm{i}$ & $11.94 \mathrm{f}$ & $27,025.42 \mathrm{f}$ & $25,226.48 f$ & $93.21 \mathrm{~b}$ & $31.82 \mathrm{~b}$ & $8,607.19 \mathrm{C}$ \\
\hline VR13-55 & $21,825.00 \mathrm{~h}$ & $3,515.83 j$ & $16.12 \mathrm{~d}$ & $23,708.25 \mathrm{~h}$ & $21,872.05 \mathrm{~g}$ & $92.32 \mathrm{~b}$ & $32.12 \mathrm{~b}$ & $7,600.45 \mathrm{~d}$ \\
\hline VR13-56 & $37,755.00 \mathrm{f}$ & $4,842.63 \mathrm{~h}$ & $12.84 \mathrm{f}$ & $24,905.87 \mathrm{~h}$ & $23,575.52 \mathrm{~g}$ & $94.69 a$ & $26.35 \mathrm{e}$ & $6,573.87 \mathrm{e}$ \\
\hline VR13-57 & $25,887.50 \mathrm{~h}$ & $6,260.11 \mathrm{~g}$ & $24.48 a$ & $24,143.45 \mathrm{~h}$ & $23,931.55 \mathrm{f}$ & $99.11 \mathrm{a}$ & $32.66 \mathrm{~b}$ & $7,880.02 \mathrm{~d}$ \\
\hline VR13-58 & $74,162.50 \mathrm{c}$ & $10,382.75 \mathrm{~d}$ & $14.00 \mathrm{e}$ & $31,355.42 \mathrm{e}$ & $31,165.10 \mathrm{~d}$ & $99.43 \mathrm{a}$ & $20.33 f$ & $6,356.60 \mathrm{e}$ \\
\hline VR13-59 & $22,800.66 \mathrm{~h}$ & 2,939.42 j & $13.10 \mathrm{e}$ & $17,017.33 j$ & $15,616.66 \mathrm{i}$ & $91.74 \mathrm{~b}$ & $21.53 \mathrm{f}$ & $3,646.98 \mathrm{~h}$ \\
\hline VR13-60 & $42,325.00 \mathrm{f}$ & $7,464.86 f$ & $17.60 \mathrm{~d}$ & $23,381.15 \mathrm{~h}$ & $21,467.82 \mathrm{~g}$ & $91.84 \mathrm{~b}$ & $28.60 \mathrm{~d}$ & $6,712.30 \mathrm{e}$ \\
\hline VR13-61 & $17,525.00 \mathrm{i}$ & $3,225.35 \mathrm{j}$ & $18.84 \mathrm{C}$ & $47,636.37 \mathrm{a}$ & $47,201.85 a$ & $99.10 \mathrm{a}$ & $18.87 \mathrm{~g}$ & $8,986.95 \mathrm{C}$ \\
\hline VR13-62 & $39,275.00 \mathrm{f}$ & $4,131.29 i$ & $10.50 \mathrm{f}$ & $48,479.55 a$ & $48,144.35 a$ & $99.30 \mathrm{a}$ & $17.42 \mathrm{~g}$ & $8,467.81 \mathrm{C}$ \\
\hline VR13-63 & $31,162.50 \mathrm{~g}$ & $4,517.60 i$ & $14.50 \mathrm{e}$ & $17,669.90 \mathrm{j}$ & $17,642.22 \mathrm{~h}$ & $99.85 \mathrm{a}$ & $30.71 \mathrm{c}$ & $5,431.95 f$ \\
\hline VR13-64 & $30,962.50 \mathrm{~g}$ & $5,095.41 \mathrm{~h}$ & $16.49 \mathrm{~d}$ & $26,414.97 \mathrm{~g}$ & $25,848.85 f$ & $97.93 a$ & $27.86 d$ & $7,367.01 \mathrm{~d}$ \\
\hline VR13-65 & $52,786.00 \mathrm{e}$ & $7,118.72 \mathrm{f}$ & $13.50 \mathrm{e}$ & $19,455.85 i$ & $18,710.71 \mathrm{~h}$ & $95.98 a$ & $26.20 \mathrm{e}$ & $5,087.47 f$ \\
\hline VR13-66 & $26,487.50 \mathrm{~h}$ & $3,600.18 \mathrm{i}$ & $13.57 \mathrm{e}$ & $28,640.12 f$ & $26,652.77 \mathrm{e}$ & $92.81 \mathrm{~b}$ & $33.03 \mathrm{~b}$ & $9,430.94 \mathrm{~b}$ \\
\hline VR13-67 & $37,275.00 \mathrm{f}$ & $5,450.74 \mathrm{~h}$ & $14.76 \mathrm{e}$ & $16,022.62 \mathrm{j}$ & $13,052.35 \mathrm{j}$ & $81.13 b$ & $32.97 \mathrm{~b}$ & $5,281.23 f$ \\
\hline VR13-68 & $24,262.50 \mathrm{~h}$ & $3,001.24 j$ & $12.43 \mathrm{f}$ & $24,671.47 \mathrm{~h}$ & $23,833.97 f$ & $96.62 a$ & $33.75 \mathrm{~b}$ & $8,330.85 \mathrm{c}$ \\
\hline VR13-69 & $31,925.00 \mathrm{~g}$ & $6,680 \cdot 13 f$ & $20.92 b$ & $27,341.75 f$ & $26,347.75 \mathrm{e}$ & $96.27 \mathrm{a}$ & $29.40 \mathrm{C}$ & $8,037.02 \mathrm{~d}$ \\
\hline VR13-70 & $19,862.50 \mathrm{i}$ & $3,380.82 j$ & $16.95 \mathrm{~d}$ & $21,612.62 \mathrm{~h}$ & $19,808.25 \mathrm{~g}$ & $91.28 \mathrm{~b}$ & $32.98 \mathrm{~b}$ & $7,139.47 \mathrm{~d}$ \\
\hline VR13-71 & $30,935.00 \mathrm{~g}$ & $3,878.91 \mathrm{i}$ & $12.52 \mathrm{f}$ & $24,412.50 \mathrm{~h}$ & $22,293.50 \mathrm{~g}$ & $91.34 \mathrm{~b}$ & $27.46 \mathrm{~d}$ & $6,721.18 \mathrm{e}$ \\
\hline VR13-72 & $29,416.00 \mathrm{~g}$ & $4,187.97 i$ & $14.50 \mathrm{e}$ & $22,023.42 \mathrm{~h}$ & $21,656.22 \mathrm{~g}$ & $98.33 a$ & $31.83 \mathrm{~b}$ & $7,009.23 d$ \\
\hline VR13-73 & $36,087.50 \mathrm{~g}$ & $5,034.79 h$ & $13.96 \mathrm{e}$ & $22,991.77 \mathrm{~h}$ & $22,048.77 \mathrm{~g}$ & $95.87 \mathrm{a}$ & $30.20 \mathrm{c}$ & $6,942.29 \mathrm{e}$ \\
\hline VR13-74 & $34,362.50 \mathrm{~g}$ & $7,711.78 \mathrm{f}$ & $22.43 a$ & $22,367.40 \mathrm{~h}$ & $22,220.37 \mathrm{~g}$ & $99.33 a$ & $28.15 d$ & $6,300.17 \mathrm{e}$ \\
\hline VR13-75 & $51,337.50 \mathrm{e}$ & $7,455.31 \mathrm{f}$ & $14.50 \mathrm{e}$ & $12,336.62 \mid$ & $10,960.50 \mathrm{j}$ & $88.40 \mathrm{~b}$ & $26.71 \mathrm{~d}$ & $3,301.40 \mathrm{~h}$ \\
\hline VR13-76 & $15,076.50 \mathrm{i}$ & $2,312.58 \mathrm{j}$ & $15.25 \mathrm{~d}$ & $26,962.12 f$ & $25,459.57 \mathrm{f}$ & $94.34 \mathrm{a}$ & $31.41 \mathrm{~b}$ & $8,471.30 \mathrm{c}$ \\
\hline VR13-77 & $15,719.50 \mathrm{i}$ & $2,823.31 \mathrm{j}$ & $17.93 \mathrm{C}$ & $8,944.12 \mathrm{~m}$ & $6,702.84 \mathrm{k}$ & $75.13 c$ & $32.02 \mathrm{~b}$ & $2,864.35 \mathrm{~h}$ \\
\hline VR13-78 & $17,482.00 i$ & $2,648.20 j$ & $15.30 \mathrm{~d}$ & $14,949.87 \mathrm{k}$ & $10,289.25 \mathrm{j}$ & $66.95 \mathrm{~d}$ & $27,75 \mathrm{~d}$ & $4,136.54 \mathrm{~g}$ \\
\hline VR13-79 & $22,175.00 \mathrm{~h}$ & $3,854.07 \mathrm{i}$ & $17.48 \mathrm{~d}$ & $12,977.70 \mid$ & $12,099.68 \mathrm{j}$ & $92.81 \mathrm{~b}$ & $29,36 \mathrm{C}$ & $3,811.15 \mathrm{~h}$ \\
\hline VR13-80 & $22,471.00 \mathrm{~h}$ & $2,962.74 j$ & $13.16 \mathrm{e}$ & $11,579.17 \mid$ & $8,574.31 \mathrm{j}$ & $74.44 \mathrm{C}$ & $30.75 c$ & $3,564.09 h$ \\
\hline VR13-81 & $33,012.50 \mathrm{~g}$ & $7,656.00 \mathrm{~h}$ & $15.49 \mathrm{~d}$ & $13,386.07 k$ & $12,061.67 j$ & $89.48 \mathrm{~b}$ & $29.50 \mathrm{C}$ & $3,962.05 \mathrm{~h}$ \\
\hline VR13-82 & $31,273.00 \mathrm{~g}$ & $6,419.49 \mathrm{~g}$ & $20.56 b$ & $2,695.47 \circ$ & 372,651 & $12.72 \mathrm{e}$ & $37.03 \mathrm{a}$ & $990.60 \mathrm{i}$ \\
\hline VR13-83 & $33,412.50 \mathrm{~g}$ & $5,695.54 \mathrm{~g}$ & $17.00 \mathrm{~d}$ & $19,963.90 \mathrm{i}$ & $18,748.07 \mathrm{~h}$ & $93.98 \mathrm{a}$ & $25.16 \mathrm{e}$ & $5,012.95 f$ \\
\hline VR13-84 & $45,475.00 \mathrm{e}$ & $6,347.20 \mathrm{~g}$ & $14.07 \mathrm{e}$ & $35,214.65 d$ & $33,125.97 d$ & $94.01 \mathrm{a}$ & $23.66 \mathrm{e}$ & $8,341.41 \mathrm{c}$ \\
\hline VR13-85 & $31,125.00 \mathrm{~g}$ & $7,331.87 \mathrm{f}$ & $23.59 a$ & $25,852.22 \mathrm{~g}$ & $24,982.22 f$ & $96.78 a$ & $27.28 d$ & $7,050.88 d$ \\
\hline VR13-86 & $29,787.50 \mathrm{~g}$ & $6,418.26 \mathrm{~g}$ & $21.53 \mathrm{~b}$ & $34,976.45 \mathrm{~d}$ & $33,943.75 d$ & $97.05 \mathrm{a}$ & $28.49 \mathrm{~d}$ & $9,967.10 \mathrm{~b}$ \\
\hline VR13-87 & $93,725.00 \mathrm{a}$ & $15,117.39 \mathrm{a}$ & $16.13 d$ & 16,810.32 j & $16,024.30 i$ & 95.26 a & $30.48 c$ & $5,138.43 f$ \\
\hline VR13-88 & $25,412.50 \mathrm{~h}$ & $4,202.27$ i & $16.50 \mathrm{~d}$ & $23,387.97 \mathrm{~h}$ & $23,364.90 \mathrm{~g}$ & $99.89 \mathrm{a}$ & $26.28 \mathrm{e}$ & $6,159.13 \mathrm{e}$ \\
\hline VR13-89 & $27,537.50 \mathrm{~g}$ & $4,609.46 \mathrm{i}$ & $16.93 d$ & $26,425.82 \mathrm{~g}$ & $25,583.02 f$ & $96.76 a$ & $35.13 a$ & $9,301.54 \mathrm{c}$ \\
\hline VR13-90 & $42,150.00 \mathrm{f}$ & $5,689.08 \mathrm{~g}$ & $13.50 \mathrm{e}$ & $19,642.85 \mathrm{i}$ & $18,378.42 \mathrm{~h}$ & $92.14 \mathrm{~b}$ & $30.42 \mathrm{c}$ & $5,994.69 \mathrm{e}$ \\
\hline VR13-91 & $33,700.00 \mathrm{~g}$ & $4,855.09 \mathrm{~h}$ & $14.46 \mathrm{e}$ & $29,116.25 \mathrm{f}$ & $28,521.95 \mathrm{e}$ & $97.96 a$ & $26.83 d$ & $7,806.30 \mathrm{~d}$ \\
\hline VR13-92 & $32,325.00 \mathrm{~g}$ & $6,341.51 \mathrm{~g}$ & $19.57 \mathrm{C}$ & $5,456.15 n$ & $4,001.77 k$ & $69.68 \mathrm{C}$ & $38.62 \mathrm{a}$ & $2,110.28 \mathrm{i}$ \\
\hline VR13-93 & $42,167.50 \mathrm{f}$ & $6,867.75 f$ & $16.28 \mathrm{~d}$ & 12,598.82। & $11,934.39 \mathrm{j}$ & $94.64 \mathrm{a}$ & $34.95 a$ & $4,402.03 \mathrm{~g}$ \\
\hline VR13-94 & $34,625.00 \mathrm{~g}$ & $4,189.62 \mathrm{i}$ & $12.10 \mathrm{f}$ & $25,912.67 \mathrm{~g}$ & $23,467.92 \mathrm{~g}$ & $90.71 \mathrm{~b}$ & 33.09 b & $8,590.48 \mathrm{c}$ \\
\hline VR13-95 & $22,012.50 \mathrm{~h}$ & $3,118.37 \mathrm{j}$ & $14.12 \mathrm{e}$ & $30,766.10 \mathrm{e}$ & $29,249.22 \mathrm{e}$ & $95.04 a$ & $29.93 \mathrm{C}$ & $9,211.45 \mathrm{c}$ \\
\hline VR13-96* & $37,208.33 f$ & $8,021.62 \mathrm{f}$ & $21.63 b$ & $32,496.70 \mathrm{e}$ & $32,141.06 \mathrm{~d}$ & $98.93 \mathrm{a}$ & $21.03 f$ & $6,849.00 \mathrm{e}$ \\
\hline VR13-97* & $51,514.66 \mathrm{e}$ & $9,375.84 \mathrm{e}$ & $18.13 \mathrm{C}$ & $27,662.57 \mathrm{f}$ & $27,196.33 \mathrm{e}$ & $98.34 \mathrm{a}$ & $24.66 \mathrm{e}$ & $6,819.07 \mathrm{e}$ \\
\hline CV (\%) & 12.17 & 11.32 & 7.61 & 7.31 & 8.75 & 4.97 & 5.79 & 10.05 \\
\hline F Value & $43.35^{* *}$ & $43.97^{* *}$ & $18.99 * *$ & $84.13^{* *}$ & $70.65^{* *}$ & $17.36^{* *}$ & $18.71^{* *}$ & $34.13^{* *}$ \\
\hline
\end{tabular}


With the modernization of many agriculture fields, breeding programs seek to obtain new cultivars with high productivity, production stability, spatial adaptability and resistance to key pests and diseases for certain crops. Thus, narrowing of the genetic basis of these materials occurs, with consequent loss of genetic diversity.

Accessions collection occurred in traditional communities from Vale do Ribeira, which, due to their traditional crop management, did not adopted modern production techniques. Therefore, it appears that this practice is one of the foundations that maintain the variability obseved in this study.

VR13-35 and VR13-87 accessions, with 97995.50 and $93725.00 \mathrm{~kg} \mathrm{ha}^{-1}$, respectively, presented the highest branch production, followed by VR13-22, with $88,250.00 \mathrm{~kg} \mathrm{ha}^{-1}$. VR13-26, VR13-70, VR13-5, VR13-21, VR13-61, VR13-78, VR13-77, VR13-76, VR13-15 and VR13-49 accessions resulted in lower branch productions per hectare.

The highest values observed in this study were higher than those found by Azevedo et al. (2015) studying 65 genotypes from the germplasm bank of UFVJM, Diamantina-MG, Brazil with values between 560 to $8140.00 \mathrm{~kg} \mathrm{ha}^{-1}$. In addition, values were also higher than those observed by Andrade Junior et al. (2014), who obtained values from 4,200 to $7,900.00 \mathrm{~kg} \mathrm{ha}^{-1}$, with seven genotypes, in Couto Magalhães-MG. However, studies conducted by Gonçalves Neto et al. (2011), in ljaci-MG, obtained higher values than the maximum observed in this study, with values of $201,550.00,236,000.00$ and $302,450.00$ $\mathrm{kg} \mathrm{ha}^{-1}$ for UFLA07-08, UFLA07-24 and UFLA07-15 accessions, respectively.

For branch dry matter production per hectare, VR13-87 accession had the best performance, with the yield of $15,117.39 \mathrm{~kg} \mathrm{ha}$ 1, followed by VR13-35 accession, with yield of $13,473.99 \mathrm{~kg} \mathrm{ha}^{-1}$. The highest means observed in this study are higher than those found by Viana et al. (2011), who observed maximum production of $9,480.00 \mathrm{~kg} \mathrm{ha}^{-1}$ for the BD-31TO genotype among eight tested genotypes, at 180 days after branches planting.

The highest branch dry matter percentages were found in VR13-57, VR13-85 and VR13-74 accessions, with values of 24.48, 23.59 and $22.43 \%$, respectively (Table 1). The observed values are similar to those found by Figueiredo et al. (2012), who observed maximum dry matter contents $(24.31 \%)$ in the branches of the BD-42 accession, in Diamantina-MG, Brazil.

Sweet potato branches and leaves is being a practive adopted by small growers to complement animal feed, after roots consumption. Sweet potato branches and leaves used animal feed supply showed promising results for dairy cows (Khalid et al., 2013), goats (Megersa et al., 2013) and pigs (Peters, 2004). Sweet potato branches good performance can be linked to its high protein content, which is between $26-33 \%$, and with considerable amounts of vitamins A, B2, $C$ and $E$ (Apata \& Babalola, 2012).

VR13-62 and VR13-61 accessions had the best root total production, with values of $48,479.55$ and $47,636.37 \mathrm{~kg} \mathrm{ha}^{-1}$, respectively (Table 1). The values are 270 and $263 \%$ higher than the Brazilian national average, respectively, which is 13,091.29 $\mathrm{kg} \mathrm{ha}^{-1}$ (FAO, 2015). VR13-82 accession obtained the lowest yield: $2,695.47 \mathrm{~kg} \mathrm{ha}^{-1}$. Therefore, the production range was of $45,784.08 \mathrm{~kg} \mathrm{ha}^{-1}$.

Values observed in this study are similar to maximum yields achieved by Azevedo et al. (2014), who obtained values of $42,070.00$ and $51,040.30 \mathrm{~kg} \mathrm{ha}^{-1}$ for BD-38 and BD-45 accessions. In addition, the values of this study are above the maximum yields observed by Azevedo et al. (2015), Massaroto et al. (2014), Cavalcante et al. (2009), Roesler et al. (2008) and Cardoso et al. (2005).

Sweet potato crop productive differences may be associated with a number of factors, such as crop season, soil type, water system, propagating material health, used genotype, plant nutrition, etc. However, accession groups with excellent yield potential are identified, and they may constitute the base population for future sweet potato genetic improvement programs.

The two commercial cultivars, Brazlândia Branca (VR13-96) and Brazlândia Roxa (VR1397), had yields of $32,496.70$ and $27,662.57 \mathrm{~kg} \mathrm{ha}^{-1}$, respectively. In studies by Azevedo et al. (2015), yields of $11,240.00 \mathrm{~kg} \mathrm{ha}^{-1}$ for the Brazlândia Roxa 
cultivar were found, in Diamantina, in the summer of $2007 / 2008$. Andrade Junior et al. (2009) found

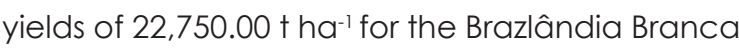
cultivar, in crops grown from December, 2005 to July, 2006.

Taking into consideration the five groupings with higher yield means by ScottKnott test, 15 accessions with yield above 30 t $\mathrm{ha}^{-1}$ were verified. Seven of them were collected in "quilombos", four in peri-urban vegetable plantations in the municipalities of Registro and Sete Barras, two in coastal communities in Pedrinhas, llha Comprida, and one in a rural garden at the municipality of Cananéia. In addition, Brazlândia Branca cultivar also presented higher means for yield.

VR13-62 and VR13-61 accessions resulted in the best commercial production performance. The five most productive accessions also achieved higher commercial productions. However, VR13-82 accession produced only $372.65 \mathrm{~kg} \mathrm{ha}^{-1}$ commercial roots (weight above $80 \mathrm{~g}$ ), i.e., only $12.72 \%$ of all commercial roots production (Table 1).

Considering roots commercialization for home consumption, which is as important as roots total production and non-commercial roots, 56 accessions were grouped with the best performance, ranging from 93.92 to $99.85 \%$ of sweet potato roots commercial production.

VR13-92, VR13-82, VR13-5, VR13-37, VR13-34, VR13-1, VR13-89, VR13-93 and VR1323 accessions presented the highest root dry matter contents, with values ranging from 34.9 to $38.62 \%$. Values were higher than those observed by Andrade Júnior et al. (2012), who evaluated 12 accessions in Diamantina-MG, in a six-month crop cycle.

VR 13-11 and VR 13-14 accessions showed the highest root dry matter yields, 12,405.39 and $12,200.19+\mathrm{ha}^{-1}$, respectively. As sweet potato dry matter consists of $85 \%$ of carbohydrates on average (Silva et al., 2008), whose main component is starch, the carbohydrate productivity above $10,000.00 \mathrm{~kg} \mathrm{ha}^{-1}$ is inferred.

Currently, in Asian countries, sweet potato has been used as starch source to produce pasta and breads. Thus, the genotype that has the best agronomic results for this purpose should be chosen.

According to Gonçalves Neto et al. (2011), most of the currently used sweet potato cultivars were selected for home consumption, and there is a huge potential for other purposes. The agronomic skills of these genotypes for other purposes need to be identified. Thus, VR1362, VR13-61, VR13-44, VR13-11 and VR13-14 accessions are promising to produce roots for home consumption; VR13-11 and VR13-14 are promising to the industry, due to their high dry matter yield per hectare; and VR13-35, VR13-87 and VR13-22 are promising to produce branches for animal feed purposes. VR13-11, VR13-22 and VR13-58 accessions are suitable for both home and animal consumption; VR13-48 is suitable for home and industry consumption (ethanol and starch); and VR13-4 and VR13-31 are suitable for animal consumption and industry.

\section{Conclusions}

It is possible to conclude that accesses VR13-62, VR13-61, VR13-44, VR13-11 and VR13-14 are promising to be produced and its roots used for home consumption; VR13-11 and VR13-14 for food industry; and VR13-35, VR13-87 and VR13-22 are promising to be grown and its branches used for animal feed purposes.

\section{Acknowledgement}

The authors thank the Sao Paulo Research Foundation (Process 2012/08763-0 and 2013/15239-9) for the financial support and the scholarship to the second author.

\section{References}

Andrade Júnior, V.C., Pereira, R.C., Dornas, M.F.S., Ribeiro, K.G., Valadares, N.R., Santos, A.A., Castro, B.M.C. 2014. Produção de silagem, composição bromatológica e capacidade fermentativa de ramas de batata-doce emurchecidas. Horticultura Brasileira 32: 91-97.

Andrade Júnior, V.C., Viana, D.J.S., Fernandes, J.S.C., Figueiredo, J.A., Nunes, U.R., Neiva, I.P. 2009. Selection of sweet potato clones for the region Alto Vale do Jequitinhonha. Horticultura Brasileira 27: 389-393.

Andrade Júnior, V.C., Viana, D.J.S., Pinto, N.A.V.D., Guimaraes, K.R., Pereira, R.C., Neiva, I.P., Azevedo, A.M., Andrade, P.C.R. 2012. Características produtivas e qualitativas de ramas e raízes de batata-doce. Horticultura 
Brasileira 30: 584-589.

Anuário Brasileiro de Hortaliças. 2015. Editora Gazeta, Santa Cruz, RS. 68 p.

Apata, D.F., Babalola, T.O. 2012. The Use of Cassava, Sweet Potato and Cocoyam, and Their By-Products by Non - Ruminants. International Journal of Food Science and Nutrition Engineering 2:54-62.

Azevedo, A.M., Andrade Júnior, V.C., Fernandes, J.S.C., Pedrosa, C.E., Oliveira, C.M. 2015. Desempenho agronômico e parâmetros genéticos em genótipos de batata-doce. Horticultura Brasileira 33: 84-90.

Azevedo, A.M., Andrade Júnior, V.C., Viana, D.J.S., Elsayed, A.Y.A.M., Pedrosa, C.E., Neiva, I.P., Figueiredo, J.A. 2014. Influence of harvest time and cultivation sites on the productivity and quality of sweet potato. Horticultura Brasileira 32: 21-27.

Cardoso, A.D., Viana, A.E.S., Ramos, P.A.S., Matsumoto, S.N., Amaral, C.L.F., Sediyama, T., Morais, O.M. 2005. Avaliação de clones de batata-doce em Vitória da Conquista. Horticultura Brasileira 23:911-914.

Cavalcante, M., Ferreira, P.V., Paixão, S.L., Costa, J.G. da, Pereira, R.G., Madalena, J.A.S. 2009. Potenciais produtivo e genético de clones de batata-doce. Acta Scientiarum. Agronomy 31: 421-426.

Cepagri - Centro de pesquisas meteorológicas e climáticas aplicadas a agricultura. 2015. A classificação climática de Köppen para o estado de São Paulo. http://www.cpa.unicamp. br/outras-informacoes/clima-dos-municipiospaulistas.html <Accessed in 20 Feb, 2015>.

Cruz, C.D. 2013. GENES - a software package for analysis in experimental statistics and quantitative genetics. Acta Scientiarum. Agronomy. 35: 271276.

EMBRAPA - Empresa Brasileira de Pesquisa Agropecuária. 2015. Batata-Doce: 'Brazlândia Branca', 'Brazlândia Roxa', 'Brazlândia Rosada' e 'Coquinho'. http://www.cnph.embrapa.br/ paginas/produtos/cultivares/batata_doce_ varias.htm <Accessed in 25 Feb, 2015>.

FAO - Fao stat data base gateway. 2015. http:// faostat3.fao.org/home/E <Access in $16 \mathrm{Feb}$. 2015>.

Figueiredo, J.A., Andrade Junior, V.C., Pereira, R.C., Ribeiro, K.G., Viana, D.J.S., Neiva, I.P. 2012. Avaliação de silagens de ramas de batatadoce. Horticultura Brasileira 30: 708-712.

Gonçalves Neto, A.C., Maluf, W.R., Gomes,
L.A.A., Gonçalves, R.J.S., SILVA, V.F. Lasmar, A. 2011. Aptidões de genótipos de batata-doce para consumo humano, produção de etanol e alimentação animal. Pesquisa Agropecuária Brasileira 46: 1513-1520.

Khalid, A.F., Elamin, K.M., Amin, A.E., Tameem, A.A.E., Mohamed, M.E., Hassan, H.E., Mohammed, M.D. 2013. Effects of using fresh sweet potato (Ipomoea batatas) vines on performance and milk yield of lactating nubian goats. Journal of Animal Science Advances 3: 226-232.

Machado, C.M.M., Abreu, F.R. 2007. Produção de álcool combustível a partir de carboidratos. Revista de Política Agrícola 15: 64-78.

Massaroto, J.A., Maluf, W.R., Gomes, L.A.A., Franco, H.D., Gasparino, C.F. 2014. Desempenho de clones de batata-doce. Ambiência 10: 73-81.

Megersa, T., Urge, M., Nurfeta, A. 2013. Effects of feeding sweet potato (Ipomoea batatas) vines as a supplement on feed intake, growth performance, digestibility and carcass characteristics of Sidama goats fed a basal diet of natural grass hay. Tropical Animal Health and Production 45: 593-601.

Monteiro, A.B., Massaroto, J.A., Gasparino, C.F., Silva, R.R., Gomes, L.A.A., Maluf, W.R., Filho, J.C.S. 2007. Silagens de cultivares e clones de batata doce para alimentação animal visando sustentabilidade da produção agrícola familiar. Revista Brasileira de Agroecologia 2: 978-981.

Monteiro, D.A., Peressin, V.A. 1977. Batata doce e cará. In: Raii, B. van; Cantarella, H., Quaggio, J.A., Furlani, A.M.C. (eds). Recomendações de adubação e calagem para o Estado de São Paulo. 2.ed. Instituto Agronômico \& Fundação IAC, Campinas, Brasil. p. 226.

Pedrosa, C.E., Andrade Júnior, V.C., Pereira, R.C., Dornas, M.F.S., Azevedo, A.M., Ferreira, M.A.M. 2015. Yield and quality of wilted sweet potato vines and its silagens. Horticultura Brasileira 33: 283-289.

Peters, D. 2004. Use of sweet potato in pig production in Asia: agricultural and socioeconomic aspects. Pig News and Information25: $25 \mathrm{~N}-34 \mathrm{~N}$.

Ramos, P.O. 2007. Futuro da ocupação na agroindústria canavieira do Brasil: uma discussão dos trabalhos disponíveis e um exercício de estimação. Informações Econômicas 37: 69-75.

Roesler, P.V.S.O., Gomes, S.D., Moro, E., Kummer, A.C.B., Cereda, M.P. 2008. Produção e qualidade de raiz tuberosa de cultivares de batata-doce no oeste do Paraná. Acta Scientiarum. Agronomy 30: 117-122. 
Vargas et al. (2016) / Agronomic characterization of sweet...

Rós, A.B., Araújo, H.S. de, Narita, N., Filho, J.T. 2011. Uso de fertilizante e tempo de permanência de mudas de batata-doce produzidas em bandejas. Pesquisa Agropecuária Brasileira 46: 845-851.

Rós, A.B., Narita, N. 2011 . Produção de mudas de batata-doce a partir de poucas plantas matrizes. Revista Brasileira de Ciências Agrárias 6: 85-89.

Silva, J.B.C., Lopes, C.A., Magalhães, J.S. 2008. A cultura da Batata-doce. Empresa Brasileira de Pesquisa Agropecuária. http:// sistemasdeproducao.cnptia.embrapa.br/ FontesHTML/Batata-doce/Batata-doce Ipomoea_batatas/composicao_uso.html <Access in 21 Feb, 2015>.

Viana, D.J.S., Andrade Júnior, V.C. de; Ribeiro, K.G., Pinto, N.A.V.D., Neiva, I.P., Figueiredo, J.A., Lemos, V.T., Pedrosa, C.E., Azevedo, A.M. 2011. Potencial de silagens de ramas de batata-doce para alimentação animal. Ciência Rural 41: 1466-1471. 